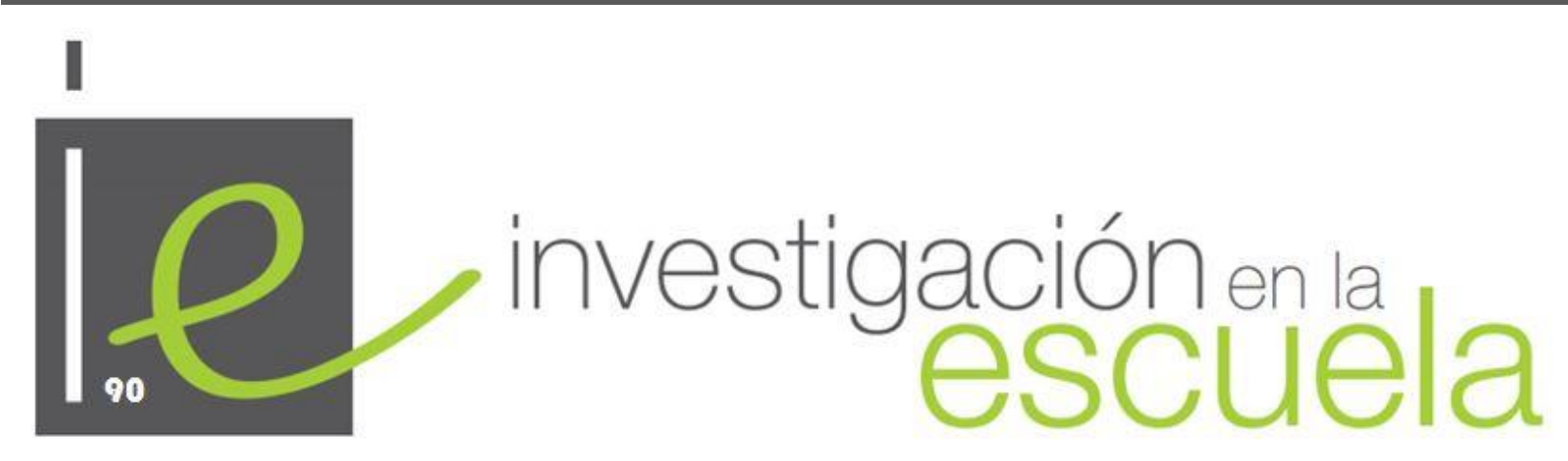

Revista académica evaluada por pares y de acceso abierto

\title{
Evaluación de la competencia lingüística a partir de un programa de desarrollo de competencias
}

\author{
Martín Pinos Quílez. \\ Centro de Innovación y Formación Educativa Juan de Lanuza (Zaragoza) \\ España
}

Citación: Pinos, M. (2016). Evaluación de la competencia lingüística a partir de un programa de desarrollo de competencias. Investigación en la Escuela, 90, 94-113. Recuperado de http://www. investigacionenlaescuela.es/articulos/R90/R90-6.pdf

Resumen: En el curso 2011-2012 se realizó una investigación evaluativa, con un enfoque metodológico complementario, sobre la aplicación de un programa educativo de desarrollo de las competencias básicas, el Proyecto Alegría, en un centro escolar de Zaragoza. Se analizaron, desde la percepción de lo usuarios, tres constructos: competencias, educación en valores y motivación del alumnado hacia el programa. Este artículo se centra en la valoración de los resultados específicos de la competencia lingüistica obtenidos en el curso escolar 2013-2014, al aplicar el programa en varios colegios públicos de Zaragoza y evaluarlo a partir de un diseño de investigación cuasi experimental. Palabras clave: "Evaluación"; "evaluación de programas"; "competencias básicas"; "competencia lingüística".

Evaluation of the linguistic competence from a program of development of competences Abstract: "Proyecto Alegría" (The Joy Project) is a project dealing with evaluative research from a complementary methodological approach about the implementation of an educational programme aimed at the development of key competences. It was first carried out during the school year 20112012 in a school in Zaragoza (Spain). In it, three constructs were analysed from the user's perspective: competences; value education; and student motivation towards the programme. 
This paper intends to provide continuity to that research, by assessing the results in linguistic competence derived from the implementation of the programme in a number of state schools in Zaragoza during the school year 2013-2014, from a quasi-experimental design.

Key words: "Assessment"; "programme assessment"; "key competences"; "linguistic competence".

\section{Évaluation des compétences linguistiques d'un programme de développement des compétences}

Resumè: Pendant l'année 2011-2012 on a effectué une recherche évaluative avec une approche méthodologique complémentaire sur la mise en œuvre d'un programme éducatif pour développer les compétences de base, le projet "Alegría"dans une école de Saragosse . Ont a analysé, depuis la perception des utilisateurs, trois éléments: les compétences, l'éducation aux valeurs et la motivation des étudiants au programme.

Cet article se centre sur l'évaluation des résultats spécifiques de la compétence lingüistique pendant l'année scolaire 2013-2014, en appliquant le programme dans plusieurs écoles publiques de Saragosse et de l'évaluer à partir d'une conception de la recherche quasi - expérimentale.

Mosts clé: "évaluation"; "évaluation de programmes"; "les compétences de base"; "maîtrise de la langue (compétence lingüistique)".

\section{Introducción}

Entre los cursos 2011-2012 y 2015-2016 evaluamos un programa educativo, el Proyecto Alegría, desde la propia percepción de los usuarios (docentes y alumnos), para constatar el grado de mejora de las competencias clave, de la educación en valores y la motivación del alumnado. El Proyecto Alegría: la abeja Berta y el abuelo Li nace en el Colegio público de educación infantil y primaria (CPEIP) María Moliner de Zaragoza en el curso 2009/2010. Un centro con un alto porcentaje de población inmigrante, superior el 50\%, con alumnado de más de 17 nacionalidades y una presencia mantenida de minorías étnicas. En el curso 2013/2014 se aplicó, además de en el CPEIP María Moliner, en dos de las cuatro aulas del CPEIP Hilarión Gimeno de Zaragoza y en la única clase de $4^{\circ}$ nivel de Educación Primaria del CPEIP Fernández Vizarra de Monzalbarba (Zaragoza). Aprovechando la circunstancia de que sólo dos de las cuatro aulas del CPEIP Hilarión Gimeno iban a aplicar el proyecto, se propuso a los tutores de los cuatro grupos participar como grupos experimentales o de control en una investigación sobre la competencia lingüística de sus alumnos, recurriendo a un diseño cuasiexperimental de pretest - postest de grupos no equivalentes, cuyos resultados centran la exposición de este artículo.

El Proyecto Alegría ${ }^{1}$ es un programa educativo de desarrollo de las competencias básicas (o clave) y la educación en valores destinado al alumnado de $4^{\circ}$ curso de Educación Primaria. Siete cuentos son el centro de interés a partir del cual se despliegan diversas tareas competenciales, incorporando los contenidos curriculares de áreas como Lengua, Matemáticas o Ciencias Naturales. La relevancia y pertinencia de esta investigación se justifica, desde nuestro punto de vista, por la abrumadora carencia de investigaciones que dirijan su atención a evaluar programas educativos orientados al desarrollo de las competencias del alumnado. Estimamos que este trabajo puede ser también relevante al facilitar al profesorado recursos avalados por la evidencia científica que le ayuden a desarrollar y evaluar las competencias, y beneficiaría al alumnado en la medida que aporta

${ }^{1}$ Visionado y descarga libre del Proyecto Alegría:

https://dl.dropboxusercontent.com/u/46039577/berta/intro.swf 
conocimiento sobre recursos que favorecerán su aprendizaje. Por similitudes de contexto, parece razonable pensar que los resultados tendrían cierta transferencia a otros centros educativos.

\section{Marco teórico}

\section{Las competencias básicas}

La incorporación de las competencias básicas en nuestro sistema educativo se gesta al amparo de la Ley Orgánica 2/2006, de 3 de mayo, de Educación (LOE).

A partir de Chomsky (1970), tanto en la lingüística como en la psicología y la educación, el concepto de competencia se vertebra en múltiples acepciones que hablan de la competencia como un comportamiento aplicado. En marzo de 2006 el MEC elabora el documento "Currículo y Competencias Básicas” adaptando al contexto español las propuestas que venían de Europa. Los contenidos de este documento se aplicarían después al Real Decreto 1513/2006, de 7 de diciembre, por el que se establecieron las Enseñanzas Mínimas correspondientes a la Educación Primaria, definiendo las ocho competencias básicas del currículo LOE. La aparición de la Ley Orgánica 8/2013, de 9 de diciembre, para la mejora de la calidad educativa (LOMCE), ha reorganizado las competencias a través del Real Decreto 126/2014, de 28 de febrero, por el que se aprueban las enseñanzas básicas del currículo de Educación Primaria; actualmente las competencias clave son siete, al haberse unido las competencias matemáticas y de mundo físico.

Tabla 1

Comparación de competencias europeas y españolas en LOE y LOMCE.

\begin{tabular}{|c|c|c|}
\hline $\begin{array}{l}\text { COMPETENCIAS CLAVE } \\
\text { EUROPA } 2006\end{array}$ & $\begin{array}{l}\text { COMPETENCIAS } \\
\text { BÁSICAS ESPAÑA } 2006\end{array}$ & $\begin{array}{l}\text { COMPETENCIAS CLAVE } \\
\text { ESPAÑA } 2014\end{array}$ \\
\hline $\begin{array}{l}\text { Comunicación en lengua } \\
\text { materna }\end{array}$ & \multirow{2}{*}{ C. lingüística } & \multirow{2}{*}{ Comunicación lingüística } \\
\hline $\begin{array}{l}\text { Comunicación en lenguas } \\
\text { extranjeras }\end{array}$ & & \\
\hline \multirow[b]{2}{*}{$\begin{array}{l}\text { C. matemática y c. en ciencias } \\
\text { y tecnología }\end{array}$} & C. matemática & \multirow[b]{2}{*}{$\begin{array}{l}\text { C. matemática y c. en ciencias y } \\
\text { tecnología }\end{array}$} \\
\hline & $\begin{array}{l}\text { C. en el conocimiento e } \\
\text { interacción con el mundo } \\
\text { físico }\end{array}$ & \\
\hline C. digital & $\begin{array}{l}\text { C. en el tratamiento de la } \\
\text { información y c. digital }\end{array}$ & C. digital \\
\hline Aprender a aprender & Aprender a aprender & Aprender a aprender \\
\hline C. sociales y cívicas & C. social y ciudadana & C. sociales y cívicas \\
\hline $\begin{array}{l}\text { Sentido de la iniciativa y el } \\
\text { espíritu de empresa }\end{array}$ & $\begin{array}{l}\text { Autonomía e iniciativa } \\
\text { personal }\end{array}$ & $\begin{array}{l}\text { Sentido de la iniciativa y el } \\
\text { espíritu emprendedor }\end{array}$ \\
\hline $\begin{array}{l}\text { Conciencia y expresión } \\
\text { culturales }\end{array}$ & C. cultural y artística & $\begin{array}{l}\text { Conciencia y expresión } \\
\text { culturales }\end{array}$ \\
\hline
\end{tabular}


En la normativa reciente, el Real Decreto 126/2014, de 28 de febrero, las competencias se definen como: "capacidades para aplicar de forma integrada los contenidos propios de cada enseñanza y etapa educativa, con el fin de lograr la realización adecuada de actividades y la resolución eficaz de problemas complejos". Además, adoptando la denominación de las competencias clave definidas por la Unión Europea, la norma considera que "las competencias clave son aquellas que todas las personas precisan para su realización y desarrollo personal, así como para la ciudadanía activa, la inclusión social y el empleo", rescatando la definición que ya en el 2006 dio la Unión Europea.

El contexto actual, caracterizado según la UNESCO (2015) por la incertidumbre y la complejidad, requiere de un concepto de competencia que refiere a la "capacidad de emplear conocimiento, entendido ampliamente como la combinación de información, comprensión, habilidades, valores y actitudes en contextos específicos y en respuesta a demandas específicas", que no únicas (Ibíd., p. 43).

La competencia en comunicación lingüística tiene un tratamiento especial en este artículo al haber sido objeto de una valoración específica de tipo cuasiexperimental, además de la valoración más cualitativa que se ha efectuado al total de las competencias. La competencia lingüística puede ser definida como "la capacidad de integrar conocimientos, habilidades y destrezas y actitudes vinculadas a la expresión y comprensión oral y escrita, para resolver problemas en diferentes situaciones y contextos" (Pinos, 2012, p.26). La actual normativa estatal nos dice que "es el resultado de la acción comunicativa dentro de prácticas sociales determinadas, en las cuales el individuo actúa con otros interlocutores y a través de textos en múltiples modalidades, formatos y soportes" (Orden ECD/65/2015, p. 6991). Es la competencia que nos dota de recursos necesarios para participar en la vida social y organizar el pensamiento a través de la lengua, interactuando a través del lenguaje en una diversidad de situaciones personales y sociales; es decir, por una parte es instrumento de comunicación oral y escrita y por otra es instrumento de construcción de conocimiento, de aprendizaje y desarrollo del pensamiento (Pérez Esteve, 2009; González, 2010). Implica hacer un uso correcto, coherente y eficaz de la lengua en función de nuestra intención y la situación en la que se aplica. Vemos que aglutina numerosos elementos a considerar; Reyzábal (2012, p.68) propone la siguiente definición:

“ (...) el conjunto de conocimientos, habilidades y destrezas que requiere el uso adecuado, correcto, coherente y estético tanto del código oral como del escrito (comprensión y expresión, análisis y síntesis, identificación, comparación, creación, recreación... de mensajes), centrándola en escuchar y hablar, leer y escribir de forma competente”.

Estamos ante una competencia transversal por naturaleza pues el lenguaje es el vehículo esencial para la construcción del pensamiento, la comunicación y la transmisión del saber, sea de la índole que sea; de ahí su importancia en la educación. En el ámbito de este artículo, la evaluación de esta competencia se ha centrado en las mismas dimensiones que las evaluaciones externas estudian: expresión escrita y comprensión oral y escrita. Es decir, no se ha tratado la expresión oral, "la gran olvidada de la enseñanza" (Millet, 2010, p.14; Briz, 2014), porque sea menos importante sino por la dificultad y coste de valorar este aspecto, y la imposibilidad de cotejar los resultados obtenidos con los resultados de pruebas oficiales.

\section{La investigación evaluativa de programas}

La investigación evaluativa puede ser entendida como un tipo de investigación aplicada "cuya meta no es el descubrimiento de conocimientos, sino valorar la aplicación de ese conocimiento." (Stufflebeam y Shinkfiel, 1987, p.19). Desde una concepción general de la 
evaluación, Rebolloso, Fernández-Ramírez y Cantón (2008, p.28) la definen como: “(...) un proceso sistemático de recoger, analizar, valorar y dar a conocer información que puede ser utilizada para cumplir con el requisito de la responsabilidad, aumentar el conocimiento disponible sobre el objeto que va a ser evaluado, o mejorar las actividades u operaciones de las intervenciones o programas".

Estaríamos ante una definición clara e ilustrativa de la evaluación como un proceso en el que el acceso, análisis y valoración de los datos abren el camino a una finalidad no ligada exclusivamente a la obtención de conocimiento sobre lo evaluado, sino también a su mejora. Estamos ante una formulación moderna que recoge la evolución de un amplio recorrido histórico en el que se han ido superando concepciones, depurando conceptos y ampliando la estrecha visión de un pasado no tan lejano. Así, por ejemplo Tyler (1950), como pionero de la evaluación educativa, basaba fundamentalmente su estrategia en la comparación entre los resultados logrados y los objetivos planteados, en un modelo de evaluación del rendimiento que ha tenido una notable influencia y que, incluso hoy día, reaparece con vigor en las propuestas de evaluación externa internacionales ligadas a PISA o el mismo modelo de evaluación por estándares de aprendizaje de la LOMCE.

De hecho, la evaluación no ha sido considerada como disciplina de conocimiento hasta el último tercio del siglo pasado, en el que aparecen guías rigurosas que prescriben cómo se ha de evaluar, fundamentalmente ligadas a la evaluación de programas, como la evaluación libre de metas (Scriven, 1973), Patton (1980), el modelo CIPP (Stufflebeam y Shinkfield, 1987), la evaluación respondente de Stake (1975) o Rossi y Freeman (1989). Recogiendo muchas de estas aportaciones y poniendo el peso en la función esencial de mejora de la evaluación, Pinos (2016, p. 262)la define como:

"Un proceso sistemático y continuo de recogida y análisis de información válida, útil, factible y diversa de un programa o de los procesos de aprendizaje y enseñanza de cara a comprenderlos y tomar decisiones que permitan mejorar la realidad evaluada, es decir, los propios programas o los mismos procesos de enseñanza y aprendizaje".

\section{Método}

Como hipótesis de trabajo partíamos de que la competencia lingüística de los alumnos que siguen el programa mejoraría significativamente respecto al alumnado que no lo trabaja. En el campo de las Ciencias de la Educación asumimos que la complejidad del objeto de estudio difícilmente puede ser explicada ni comprendida en toda su extensión desde un único paradigma, por lo que es conveniente la complementariedad epistemológica de, en términos de Popkewitz (1989), los paradigmas o ciencias analítica y simbólica. El diseño cuasiexperimental que complementó la investigación nos sitúa en la línea del paradigma positivista y de diseños ligados a una perspectiva tradicional de la evaluación defendida, por ejemplo, por Campbell y Stanley (1970) que pone el énfasis en la medida y los valores cuantitativos. Reconocemos que un diseño de este tipo plantea una visión reduccionista de la evaluación si no fuera porque, como se ha explicado, este diseño cuasiexperimental lo que hace es enriquecer las aportaciones cualitativas que a través de otros instrumentos y fuentes se han hecho del programa.

En el CPEIP Hilarión Gimeno, en el curso 2013/2014, se distinguió entre grupos experimentales y de control para abordar un diseño cuasiexperimental pretest-postest con grupo de control no equivalente para la evaluación de la competencia lingüística; es decir un diseño de grupo de control no equivalente en la tipología de Stanley y Campbell (1995). Se trabajó el programa con los grupos experimentales y grupos de control no equivalentes, no sometidos al programa, para contrastar resultados. Al decir "no equivalentes" nos referimos a que no poseían equivalencia preexperimental de muestreo (Ibíd., p.93), o sea, a la obligada falta de aleatoriedad al construir los 
grupos dado que nos venían impuestos, dentro del mismo colegio, por la conformación natural de grupos-aula y por la decisión personal, y ajena al investigador, de ciertos docentes sobre su interés o no por aplicar el proyecto. No obstante, se buscó controlar esta circunstancia aplicando un pretest en competencia lingüística al principio del curso para comprobar que no eran significativamente distintos. Y luego, al final del curso, un postest para comprobar el grado de evolución y, en su caso, las diferencias de resultados entre grupos en función de las diferencias iniciales.

A priori, se consideran las siguientes estrategias para el control de amenazas a la validez interna o credibilidad y a la validez externa o transferibilidad de la investigación:

- Control de la amenazas a la credibilidad: el sesgo de instrumentación, para dar consistencia a los instrumentos elegidos, se ha intentado corregir recurriendo a la validación por expertos y a pruebas bien contrastadas. La aplicación/valoración de las pruebas ha sido realizada por un mismo investigador para asegurar idénticos criterios de aplicación y evaluación, recurriendo finalmente a la multiinstrumentalidad y a la triangulación de fuentes de datos (Berardi, 2016).

- Control de las amenazas a la transferibilidad: somos conscientes de que en una investigación de esta naturaleza, y centrada en casos particulares, las generalizaciones de tipo racionalista, sin ser posibles, han de admitir cierta transferencia entre contextos similares. En el CPEIP Fernández Vizarra, al coincidir la muestra con el total de la población objeto de estudio (el alumnado de $4^{\circ}$ nivel de Educación Primaria), aseguramos la validez poblacional. En el CPEIP Hilarión Gimeno la distribución de grupos experimentales y de control según las aulas naturales, sin intervención alguna sobre ellas, y la aplicación de pretest y postest deberían compensar este sesgo.

Al realizarse la investigación en su contexto natural avalamos la validez ecológica (Bronfenbrenner, 1987). Por otro lado, el sesgo de reactividad (Behar y Riba, 1993), por el que los sujetos al sentirse evaluados se comportan de forma distinta, no es razonable atribuirlo con niños de esta edad ( 9 años) ante la variable investigada.

\section{Determinación de la población y de la muestra}

Se consideraron como informantes clave a la práctica totalidad de los alumnos de $4^{\circ}$ nivel de Educación Primaria del CPEIP Hilarión Gimeno y del CPEIP Fernández Vizarra (curso escolar 2013/2014). En el CPEIP Hilarión Gimeno, al contar con cuatro líneas, se constituyeron dos grupos experimentales y dos de control respetando los grupos naturales de clase. El CPEIP Fernández Vizarra cuenta con una sola línea así que el único grupo de $4^{\circ}$ nivel de Educación Primaria participó también en esta investigación como grupo experimental, una vez comprobado, a partir del pretest, que no había diferencias significativas con los alumnos del otro colegio.

\section{Instrumentos de recogida de la información}

Siendo consecuentes con la complementariedad metodológica ya expuesta, se consideró importante, por un lado, la "multiinstrumentalidad" (cuestionarios, entrevistas semiestructuradas, grupos de discusión, análisis documental y pruebas de competencia lingüística), afrontando la obtención de datos sobre las variables dependientes consideradas (competencias en general y competencia lingüística) con diferentes instrumentos, y por otro lado la triangulación de técnicas de recogida de datos para contrastar la información recabada.

El CPEIP Hilarión Gimeno, en el curso 2013/2014, nos brindó una excelente oportunidad al disponer de 4 grupos aula naturales, y 2 tutores manifiestamente interesados en probar el programa; los grupos de los otros 2 tutores servirían de grupos de control. Con el objeto de medir la 
evolución del nivel del alumnado de $4^{\circ}$ de Primaria en la competencia lingüística en lengua castellana se decidió aplicar un pretest en septiembre de 2013, al principio del curso, y un postest en mayo de 2014 a los grupos de control y a los grupos experimentales. Para el postest el uso de una de las pruebas oficiales ya aplicadas en Aragón parecía una solución idónea al tratarse de test rigurosamente elaborados, pilotados en una amplia población y que cuentan además con amplia información sobre los resultados obtenidos en la comunidad autónoma. Se optó así por la última prueba aplicada hasta este momento, la del curso escolar $2011 / 2012^{2}$. La prueba que se usó como pretest se construyó a partir de la aplicada en Aragón en la evaluación censal de diagnóstico del curso escolar 2008/2009³. Se adaptaron o sustituyeron algunos contextos e ítems para adecuarlo al nivel de alumnado de $4^{\circ}$ de educación primaria en su inicio de curso (nivel curricular de $3^{\circ}$ de primaria).

\section{Análisis de datos}

Utilizando el programa de tratamiento de datos cuantitativos SPSS en su versión 19.0, respecto a la competencia lingǘstica, teniendo en cuenta los resultados brutos, es decir la puntuación total obtenida en el pretest y en el postest por cada niño, se analizó la normalidad en cada una de las variables para determinar si podían aplicarse pruebas paramétricas. Tras haber aceptado que en el estado previo a la intervención tanto el grupo control como el experimental seguían distribuciones normales se realizó una prueba T de Student para comprobar que sus medias, en la variable puntuación total en el Pretest, no eran estadísticamente distintas de modo significativo.

$\mathrm{Al}$ no pasar el test de normalidad el grupo experimental de la segunda variable, puntuación total en el Postest, analizamos las diferencias utilizando una prueba no paramétrica: la U de MannWhitney. Mann-Whitney comprueba a través de una distribución de rangos si los promedios son iguales en ambas distribuciones. La prueba de Kolmogorov-Smirnow para dos muestras se realizó como análisis adicional de igualdad de distribuciones.

Se recurrió también al análisis de los descriptivos de las variables para comprobar si existía o no solapamiento en los intervalos de confianza. Utilizando la valoración según la Teoría de Respuesta a los Ítems (TRI) correspondiente a la misma prueba que se pasó en 2012 en Aragón, se procedió al análisis de estadísticos descriptivos del postest en los dos grupos de estudio. Ampliamos la comparación con la media correspondiente a los resultados del CPEIP Hilarión Gimeno en la prueba censal del 2012 (526,88), como grupo de control añadido, puesto que su alumnado de $4^{\circ}$ de Educación Primaria realizó la misma prueba usada en esta investigación como postest en el 2014, dos años antes.

Para completar el análisis se realizaron tres comparaciones a partir de T de Student: resultados del Grupo de control 2012 vs Grupo experimental, resultados del Grupo de control 2012 vs Grupo de control 2014 y resultados del Grupo experimental vs Grupo de control 2014. Determinado en su caso el coeficiente de correlación biserial-puntual, se calculó la proporción de la varianza debida a pertenecer al grupo experimental y el tamaño del efecto según Cohen y Hedges. Para ello calculamos la diferencia tipificada que nos permitió, en términos de desviaciones típicas, establecer la magnitud de la diferencia entre los resultados de los grupos analizados.

Ahondando todavía un poco más en el tamaño del efecto, pasamos la prueba de rangos con signo de Wilcoxon (no paramétrica pero con una baja probabilidad de cometer el error de aceptar la hipótesis nula cuando es falsa) al grupo experimental y al grupo de control para obtener información sobre si

\footnotetext{
2 Ver Anexo VII Postest de Competencia en comunicación lingüística y cuadernillo de corrección en: https://zaguan.unizar.es/record/57707/files/TUZ_0957_pinos_evaluacion.pdf

${ }^{3}$ Ver Anexo VI Pretest de Competencia en comunicación lingüística y cuadernillo de corrección en: https://zaguan.unizar.es/record/57707/files/TUZ_0957_pinos_evaluacion.pdf
} 
había alguna variación significativa en la distribución o medida de tendencia central entre la puntuación en el pretest y el postest.

\section{Resultados}

Para la variable puntuación total en el Pretest, asumiendo igualdad de las varianzas (ya que el p-valor es 0,473), la prueba T de Student de comparación de medias (equivalente a una ANOVA de efectos fijos de un solo factor con dos niveles) arroja un p-valor de 0,657 , por lo que podemos concluir que las diferencias no son significativas, o sea aceptamos la hipótesis nula que consiste en suponer que las medias no difieren significativamente en el estado de preintervención.

Tabla 2.

Prueba de muestras independientes respecto a los resultados del pretest.

\begin{tabular}{|c|c|c|c|c|c|c|c|c|}
\hline \multicolumn{8}{|c|}{ Prueba de muestras independientes } \\
\hline \multicolumn{2}{|c|}{ Prueba de Levene para la igualdad de } \\
varianzas
\end{tabular}

Para la segunda variable, puntuación total en el Postest, analizamos las diferencias utilizando la prueba no paramétrica: U de Mann-Whitney. 
Tabla 3

Diferencia de resultados en el postest.

\begin{tabular}{|c|c|c|c|c|c|}
\hline \multicolumn{6}{|c|}{ Rangos } \\
\hline \multicolumn{3}{|c|}{ Tipo de grupo (Ctrl o Exper) } & $\mathrm{N}$ & $\begin{array}{l}\text { Rango } \\
\text { promedio }\end{array}$ & Suma de rangos \\
\hline \multirow{3}{*}{ Postest } & \multicolumn{2}{|c|}{ Gr de Control } & 44 & 43,02 & 1893,00 \\
\hline & \multicolumn{2}{|c|}{ Gr Experimental } & 64 & 62,39 & 3993,00 \\
\hline & \multicolumn{2}{|c|}{ Total } & 108 & & \\
\hline \multicolumn{3}{|c|}{ Test Statistics ${ }^{\mathrm{a}}$} & & & \\
\hline \multirow{2}{*}{\multicolumn{2}{|c|}{$\begin{array}{l}\text { Mann-Whitney } \\
\text { U }\end{array}$}} & Postest & & & \\
\hline & & 903,000 & & & \\
\hline \multicolumn{2}{|c|}{ Wilcoxon W } & 1893,000 & & & \\
\hline \multicolumn{2}{|l|}{$\mathrm{Z}$} & $-3,164$ & & & \\
\hline \multicolumn{2}{|c|}{$\begin{array}{l}\text { Asymp. Sig. (2- } \\
\text { tailed) }\end{array}$} & ,002 & & & \\
\hline \multicolumn{3}{|c|}{$\begin{array}{l}\text { a. Variable grupo: Tipo de grupo } \\
\text { (Ctrl o Exper) }\end{array}$} & & & \\
\hline
\end{tabular}

El p-valor para esta prueba, 0,002, permitió concluir que había diferencias significativas y el grupo experimental había obtenido en el postest resultados significativamente mejores que el de control. Sobre una puntuación máxima del postest situada en 30 puntos, la Figura 1 exhibe las medias de resultados anunciando diferencias relevantes, en este caso a favor del grupo experimental.

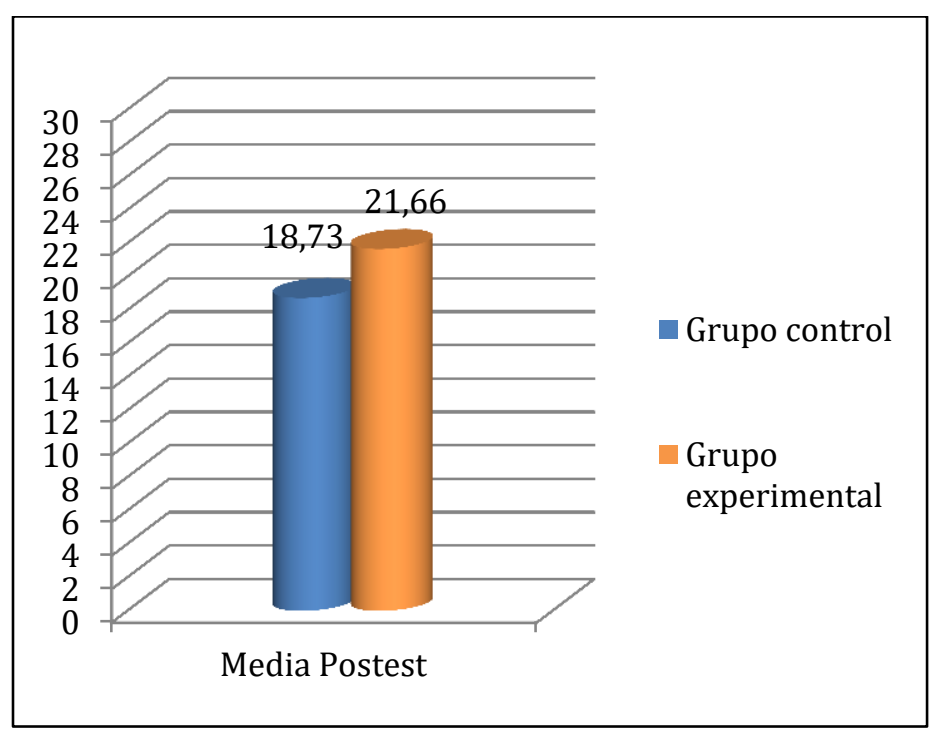

Figura 1. Comparación de medias de las puntuaciones obtenidas en el postest.

La prueba de Kolmogorov-Smirnow para dos muestras arrojó un p-valor de 0,006 que lleva a rechazar la hipótesis nula de igualdad de distribuciones confirmando la conclusión extraída con Mann- Whitney. 
$\mathrm{Al}$ analizar los descriptivos de las variables observamos cómo en la variable puntuación total en el Postest apenas existe solapamiento en los intervalos de confianza, lo que corrobora la conclusión en cuanto a la diferencia significativa de las medias.

Tabla 4

Estadísticos descriptivos.

\begin{tabular}{|c|c|c|c|c|c|c|c|c|c|c|c|}
\hline \multicolumn{12}{|c|}{ Descriptivos } \\
\hline & \multicolumn{3}{|c|}{$\begin{array}{c}\text { Tipo de grupo (Control o } \\
\text { Experimental) }\end{array}$} & Estadíst. & $\begin{array}{l}\text { Error } \\
\text { típico }\end{array}$ & & \multicolumn{3}{|c|}{$\begin{array}{c}\text { Tipo de grupo (Control o } \\
\text { Experimental) }\end{array}$} & Estad. & $\begin{array}{l}\text { Error } \\
\text { típico }\end{array}$ \\
\hline \multirow{6}{*}{ 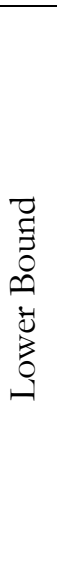 } & \multirow{3}{*}{$\begin{array}{l}\overrightarrow{0} \\
\stackrel{\Xi}{\Xi} \\
0 \\
\dot{0} \\
\dot{U}\end{array}$} & \multicolumn{2}{|l|}{ Media } & 14.86 & .869 & \multirow{6}{*}{ 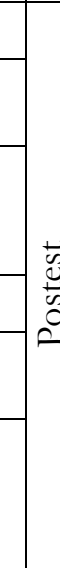 } & \multirow{3}{*}{$\begin{array}{c}\overrightarrow{0} \\
\stackrel{\overrightarrow{0}}{0} \\
0 \\
\dot{0} \\
\dot{0}\end{array}$} & \multicolumn{2}{|l|}{ Media } & 18.73 & .789 \\
\hline & & \multirow{2}{*}{$\begin{array}{l}95 \% \\
\text { Intervalo } \\
\text { confianza } \\
\text { de la } \\
\text { media }\end{array}$} & $\begin{array}{l}\text { Banda } \\
\text { inferior }\end{array}$ & 13.11 & & & & \multirow{2}{*}{$\begin{array}{l}95 \% \\
\text { Intervalo de } \\
\text { confianza } \\
\text { de la media }\end{array}$} & $\begin{array}{l}\text { Banda } \\
\text { inferior }\end{array}$ & 17.14 & \\
\hline & & & $\begin{array}{l}\text { Banda } \\
\text { superior }\end{array}$ & 16.62 & & & & & $\begin{array}{l}\text { Banda } \\
\text { superior }\end{array}$ & 20.32 & \\
\hline & 표 & \multicolumn{2}{|l|}{ Media } & 14.39 & .649 & & \multirow{3}{*}{ 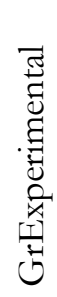 } & \multicolumn{2}{|l|}{ Media } & 21.66 & .743 \\
\hline & : & \multirow{2}{*}{$\begin{array}{l}95 \% \\
\text { Intervalo } \\
\text { confianz } \\
\text { a de la } \\
\text { media }\end{array}$} & $\begin{array}{l}\text { Banda } \\
\text { inferior }\end{array}$ & 13.09 & & & & \multirow[b]{2}{*}{$\begin{array}{l}\text { lo de } \\
\text { zza } \\
\text { edia }\end{array}$} & $\begin{array}{l}\text { Banda } \\
\text { inferior }\end{array}$ & 20.17 & \\
\hline & 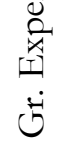 & & $\begin{array}{l}\text { Banda } \\
\text { superior }\end{array}$ & 15.69 & & & & & $\begin{array}{l}\text { Banda } \\
\text { superior }\end{array}$ & 23.14 & \\
\hline
\end{tabular}

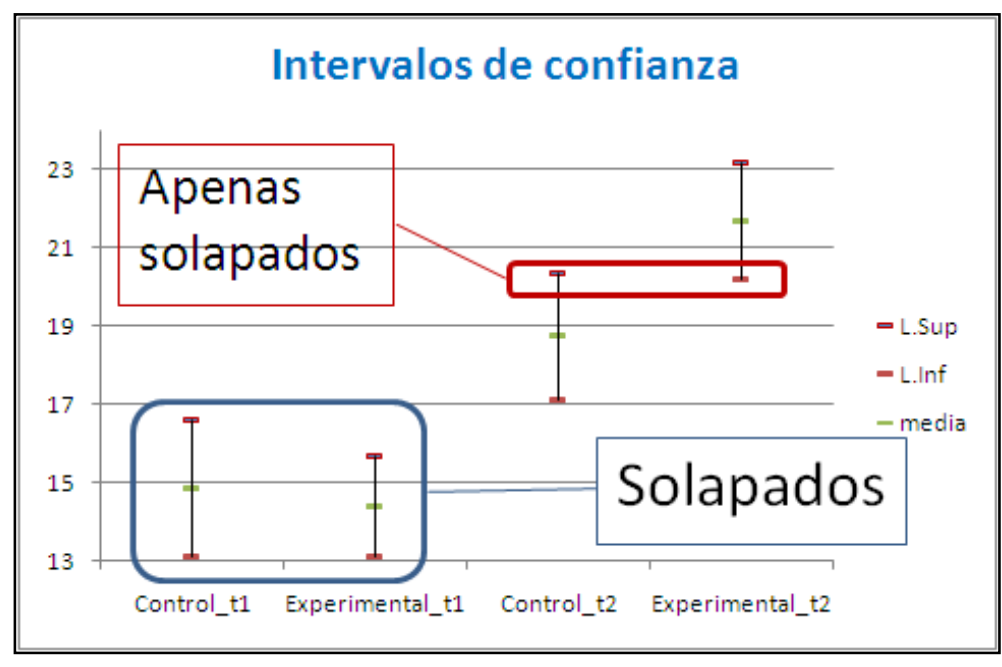

Figura 2. Intervalos de confianza.

Desde la Teoría de Respuesta a los Ítems (TRI) también se obtiene una diferencia significativa en cuanto a los resultados obtenidos por los alumnos. 
Tabla 5

Estadísticos descriptivos aplicables al Postest según la TRI.

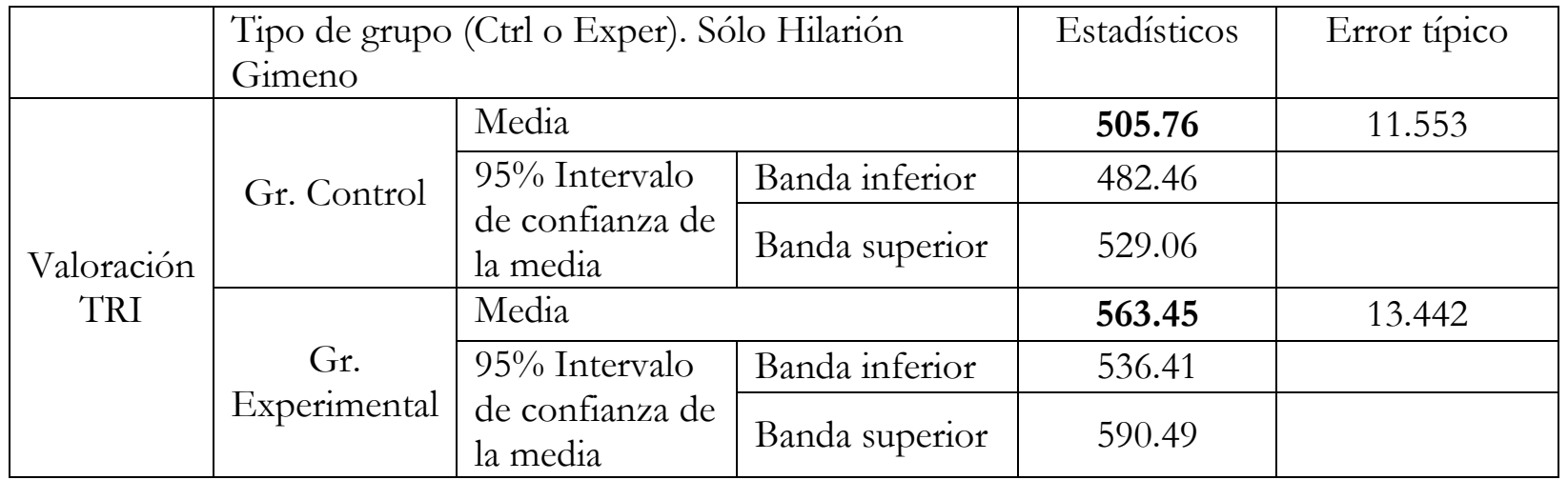

La comparación, como se adivina en la Figura 3, con la media correspondiente a los resultados del CPEIP Hilarión Gimeno en la prueba censal del 2012 (526,88), como grupo de control añadido, se trasladó también a los intervalos de confianza que se representan en la Figura 4.

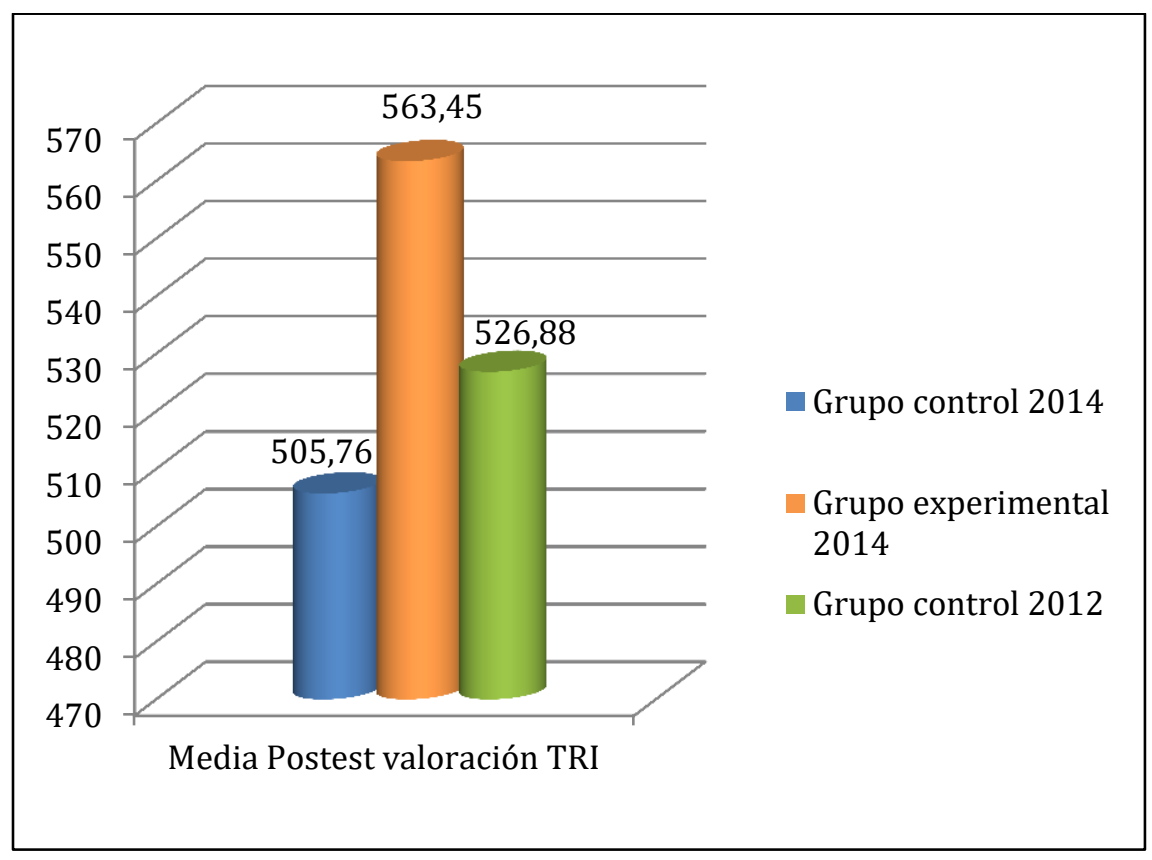

Figura 3. Media postest a partir de la TRI.

Se observa que no hay solapamiento entre los intervalos de confianza del grupo de control y el experimental, lo que nos permite concluir que, también utilizando la valoración TRI, es significativamente mejor el resultado del grupo experimental. 


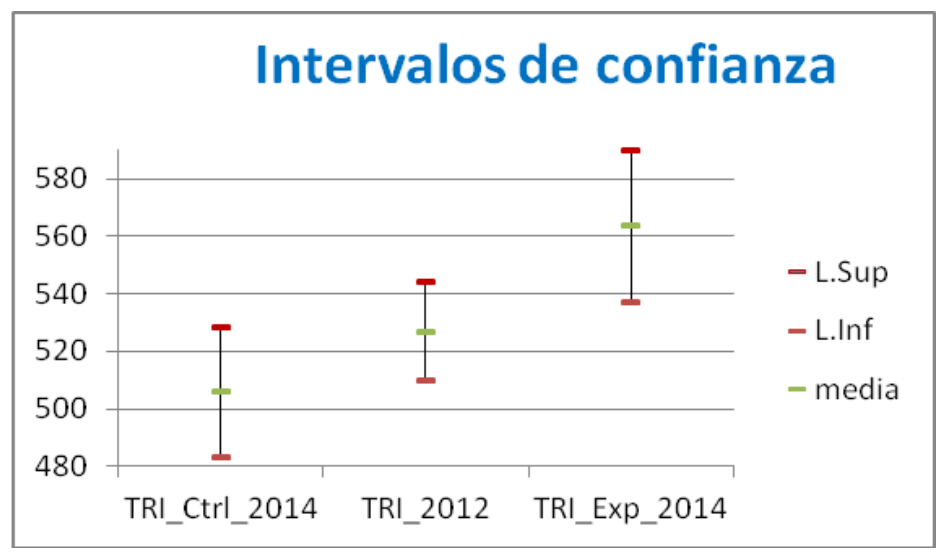

Figura 4. Intervalos de confianza.

A continuación se muestran los resultados del Grupo de control 2012 vs Grupo experimental, los resultados del Grupo de control 2012 vs Grupo de control 2014 y los resultados del Grupo experimental vs Grupo de control 2014.

Resultados 2012 vs Grupo experimental. El p-valor indica que las diferencias son estadísticamente significativas (Tabla 6).

Tabla 6

T de Student Grupo 2012 vs Grupo Experimental 2014.

\begin{tabular}{|c|c|c|c|}
\hline Grupo & Resultados 2012 & Experimental & \\
\hline Test & & Post & \\
\hline Tamaño muestra & 75 & 48 & \\
\hline Media & 526.88 & 563.449 & \\
\hline \multicolumn{3}{|l|}{ Desviación típica } & \\
\hline Poblacional & 75.0472 & 92.1558 & \\
\hline Muestral & 75.5526 & 93.131 & \\
\hline $\mathrm{t}($ Student $)=$ & 2.3887 & $\mathrm{p}=$ & 0.0185 \\
\hline
\end{tabular}

Tabla 7.

Coeficiente de correlación biserial-puntual.

\begin{tabular}{|r|l|l|l|l|}
\hline \multicolumn{6}{|c|}{ Coeficiente de correlación biserial-puntual } & \\
\hline $\mathrm{r}_{\mathrm{bp}}=$ & 0.2122 & & & \\
\hline $\mathrm{r}_{\mathrm{bp}}{ }^{\wedge} 2=$ & 0.0450 & $\rightarrow$ & 4.5032 & $\%$ de la varianza \\
\hline
\end{tabular}

La proporción de la varianza debida a pertenecer al grupo experimental es de 4,50\%. Y el tamaño del efecto: 
Tabla 8

Tamaño del efecto Grupo 2012 vs Grupo experimental 2014.

\begin{tabular}{|c|c|c|c|c|}
\hline \multicolumn{6}{|c|}{ Tamaño del efecto (effect size) } \\
\hline Cohen & $\sigma=$ & 82.1488 & $\mathrm{~d}=$ & 0.4452 \\
\hline Hedges & $\sigma=$ & 82.8249 & $\mathrm{~g}=$ & 0.4415 \\
\hline
\end{tabular}

Tomando el de Cohen, interpretaríamos que la media correspondiente al grupo experimental supera en 0.4452 veces una desviación típica al grupo de 2012 en la prueba realizada. En términos de percentiles, y teniendo en cuenta la probabilidad correspondiente a este valor en la normal tipificada $\mathrm{P}(\mathrm{Z}<0.4452)=0.6719$, estaríamos diciendo que un alumno medio del grupo experimental tendría un percentil de 67.19 en el grupo de control, es decir supera casi al 70\% de los alumnos del grupo de control. Dicho de otra forma, un estudiante medio del grupo experimental supera a un 17\% más de alumnos si lo incluimos en el grupo de control.

Resultados 2012 vs Grupo de control. El p-valor $(0,1458)$ indica que las diferencias no son estadísticamente significativas, como era de esperar pues ninguno de los grupos se había sometido a la intervención.

Tabla 9

T de Student Grupo 2012 vs Grupo Control 2014.

\begin{tabular}{|c|c|c|}
\hline Grupo & Resultados 2012 & Control \\
\hline Test & & Post \\
\hline Tamaño muestra & 75 & 44 \\
\hline Media & 526.8796 & 505.7623 \\
\hline Desviación típica & & \\
\hline Poblacional & 75.0472 & 75.7608 \\
\hline Muestral & 75.5526 & 76.6367 \\
\hline $\mathrm{t}($ Studen $\mathrm{t})=$ & 1.4641 & $\mathrm{p}=$ \\
\hline
\end{tabular}

Grupo experimental vs Grupo de control 2014. El p-valor $(0,0017)$ indica que las diferencias son estadísticamente significativas.

Tabla 10

T de Student Grupo Control vs Grupo Experimental.

\begin{tabular}{|c|c|c|c|}
\hline Grupo & Control & Experimental & \\
\hline Test & Post & Post & \\
\hline Tamaño muestra & 44 & 48 & \\
\hline Media & 505.7623 & 563.4495 & \\
\hline \multicolumn{3}{|l|}{ Desviación típica } & \\
\hline Poblacional & 75.7608 & 92.1558 & \\
\hline Muestral & 76.6367 & 93.1310 & \\
\hline $\mathrm{t}($ Student $)=$ & 3.2271 & $\mathrm{p}=$ & 0.0017 \\
\hline
\end{tabular}


Tabla 11

Coeficiente de correlación biserial-puntual.

\begin{tabular}{|r|c|c|c|c|}
\hline \multicolumn{5}{|c|}{ Coeficiente de correlación biserial-puntual } \\
\hline $\mathrm{r}_{\mathrm{bp}}=$ & 0.322 & & & \\
\hline $\mathrm{r}_{\mathrm{bp}}{ }^{\wedge} 2=$ & 0.1037 & $\rightarrow$ & 10.371 & $\%$ de la varianza \\
\hline
\end{tabular}

La proporción de la varianza debida a pertenecer al grupo experimental es de 10,37\%. Y el tamaño del efecto se puede consultar en la Tabla 12.

Tabla 12

Tamaño del efecto.

\begin{tabular}{|l|r|r|r|r|}
\hline \multicolumn{6}{|c|}{ Tamaño del efecto (effect size) } \\
\hline Cohen & $\sigma=$ & 84.7115 & $\mathrm{~d}=$ & 0.6810 \\
\hline Hedges & $\sigma=$ & 85.6476 & $\mathrm{~g}=$ & 0.6735 \\
\hline
\end{tabular}

Tomando el de Cohen, interpretaríamos que la media correspondiente al grupo experimental supera en 0.6810 veces una desviación típica al grupo de control en la prueba realizada. En términos de percentiles, y teniendo en cuenta la probabilidad correspondiente a este valor en la normal tipificada $\mathrm{P}(\mathrm{Z}<0.6810)=07521$, estaríamos diciendo que un alumno medio del grupo experimental tendría un percentil de 75.21 en el grupo de control, es decir supera al 75\% de los alumnos del grupo de control. Dicho de otra forma, un estudiante medio del grupo experimental supera a un 25\% más de alumnos si lo incluimos en el grupo de control confirmando los resultados antes vistos.

\section{Conclusiones}

El rigor científico no puede ir separado de la dimensión ética de investigación. Somos conscientes de que el proceso de evaluación desarrollado, el análisis de los datos y su interpretación, se halla mediado, y es honesto reconocerlo, por nuestra propia cosmovisión del mundo, nuestros valores y creencias. Admitir esto como premisa de partida intenta poner en su justo valor las conclusiones y el esfuerzo por la cientificidad perseguida en el diseño y su aplicación.

Respecto a la hipótesis de trabajo, "La competencia lingüística de los alumnos que siguen el programa mejorará significativamente respecto al alumnado que no lo trabaja", la investigación nos confirma que en los casos estudiados el programa resulta de utilidad para promover mejoras en el desarrollo de la competencia lingüística aportando una herramienta eficaz al profesorado para su trabajo en el aula. Mediante un cuestionario de percepción del grado de consecución de los objetivos del programa se preguntó a los docentes sobre las cuatro grandes dimensiones de la competencia lingüística. El desarrollo de la comprensión oral y escrita se valoró con una media de 4,60 sobre 5, la expresión oral con 4.30 y la expresión escrita con 4,40. Son datos a considerar pero no aportan información respecto a poder comparar con alumnos que no han realizado el programa, tal y como señala en la hipótesis de trabajo. Los resultados mostrados por el diseño cuasiexperimental entre el grupo experimental y el grupo de control en los diferentes análisis llevados a cabo, sí evidencian mejoras significativas en la variable investigada. La comparación con respecto a la cohorte del 2012, que realizó dos años antes la misma prueba que el grupo experimental, también muestra que los resultados de los alumnos que han seguido el programa son significativamente mejores, apoyando la hipótesis de partida. El tamaño del efecto, habida cuenta de que el proyecto Alegría no es un 
programa específico de competencia lingüística y que los tiempos dedicados al mismo son ciertamente limitados, se muestra muy positivo.

Respecto al propio diseño de investigación, resulta relevante reseñar la potencialidad del modelo CIPP de Stufflebeam como sistema evaluativo a la hora de determinar, organizar, contrastar y validar informaciones procedentes de diversas fuentes e instrumentos cuantitativos y cualitativos como ocurre en la investigación en la que se enmarca este trabajo. Entre otros motivos porque determina con claridad las fases clave del programa y en qué momentos han de ser evaluadas. Esta parte de la investigación, centrada específicamente en la fase de resultados, aporta información válida para triangular con otras fuentes y constatar que el programa responde a las necesidades del contexto en el que está inmersa la escuela, cuenta en su planificación y estructura con los elementos necesarios para su funcionamiento idóneo y ha obtenido unos resultados valorados muy positivamente por los implicados en el mismo.

El diseño cuasiexperimental elegido de prestest y postest en un ámbito educacional, para la variable estudiada, se ha mostrado muy idóneo dada la obligada ausencia de aleatorización en la formación de los grupos de investigación. Comprobado mediante el análisis del pretest que los grupos control y experimental no mostraban diferencias significativas, este tipo de diseño, a partir de los resultados del postest, nos ha permitido probar una relación causal entre la variable independiente y la variable dependiente y mostrar la eficacia y efectividad del programa evaluado.

\section{Limitaciones y futuras líneas de investigación}

En la línea definida en esta investigación, este trabajo ha proporcionado un análisis y resultados ciertamente positivos, no exentos de aspectos mejorables, que contribuirán, sin duda, al perfeccionamiento del programa educativo. Pero es necesario destacar que estos resultados han de ser interpretados con la debida cautela. Sin duda, este estudio cuenta con limitaciones entre las que señalaríamos que la investigación se ha focalizado en un estudio de caso (centrado en algunos colegios) y, por tanto, en una realidad concreta, por lo que es probable que en otros contextos, con alumnos y profesores diferentes, los resultados fueran distintos. No obstante, desde el rigor que nos proporciona el diseño cuasiexperimental elegido, los resultados pueden ser ilustrativos y contribuir a mostrar estrategias de desarrollo de la competencia lingüística de forma globalizada a partir de tareas competenciales, al menos para colegios de características similares a las de los que nos ocupan.

En el ámbito aplicado, creemos que es necesario crear más programas educativos sustentados en las competencias y, acto seguido, realizar más estudios contrastados de los mismos haciendo un seguimiento de su aplicación real para poder determinar cómo funcionan, para quiénes funcionan y qué los hace efectivos, trasladando así los resultados a la comunidad educativa y científica para mejorar futuros proyectos. En el momento de elaborar este trabajo no tenemos constancia de programas similares aplicados en España con los que podamos comparar y hacer una reflexión sobre los resultados obtenidos pero, sin duda, estudios comparativos de este tipo aportarían una interesante línea de trabajo.

Es sensato reconocer que a pesar de los cambios y avances manifestados por el alumnado, la consolidación y profundización de estos cambios requerirá sin embargo, lógicamente, una acción más consistente y continuada que tenga reflejo en las acciones educativas que se llevan a cabo en el aula al margen del programa.

Una posible mejora de esta investigación podría estar en la ampliación de la fase cuasiexperimental con grupos experimentales y grupos de control a otras competencias también trabajadas en el programa, como son la matemática, la científica o las competencias sociales y cívicas. La extensión del programa a centros urbanos y rurales con contextos diferenciados ofrecería un panorama ilustrativo de buena parte de la variedad de centros educativos que existen en Aragón y 
España, y con ello establecer un análisis comparativo diferenciando, por ejemplo, entre colegios urbanos y rurales, entre centros con alumnos procedentes mayoritariamente de familias de nivel sociocultural medio-bajo y centros con alumnado de familias de clase media, centros con alumnado multiétnico y centros con alumnado principalmente autóctono, centros públicos y centros concertados.

El haber considerado el tamaño del efecto, en los análisis de los resultados, abre una futura línea de investigación diferenciando varios grupos experimentales en los que se prueben metodologías diferentes para la confección de las mismas tareas competenciales (por ejemplo trabajo individual, trabajo en parejas, grupos cooperativos, grupos interactivos, etc.) y determinar a partir de los resultados cuáles han tenido mayor impacto.

Las opciones, los interrogantes, las vías que se abren son muchas pero quisiéramos ir concluyendo con la consideración de que a raíz de los resultados de esta investigación y en el marco estudiado el proyecto Alegría: La abeja Berta y el abuelo Li, puede considerarse un recurso didáctico útil y válido para mejorar la formación del alumnado y para ayudar al profesorado a desarrollar la competencia lingüística en el aula, en $4^{\circ}$ nivel de la Educación Primaria; así como que los resultados sugieren líneas de avance y mejora que sin duda podrán contribuir a perfeccionar el programa y a establecer un punto de partida más consistente y riguroso para construir futuros programas en otros niveles educativos que sean útiles a las finalidades de la educación. Finalidades que si queremos que no se alejen de la mejora de la condición humana y la sociedad deberán contemplar el pleno desarrollo de la personalidad del niño y su felicidad, más allá del desarrollo focalizado de una u otra competencia clave.

\section{Referencias}

Behar, J. y Riba, C. (1993). Sesgos de la observación. En M.T. Anguera (Ed.), Metodología Observacional en la investigación psicológica (27-128), Vol. 2. Barcelona: PPU.

Berardi, L. (2016). Multimétodos. Un recurso para potenciar hallazgos en investigación social. En Abero, L., Berardi, L., Capocasale, A., García, S. y Rojas, R. Investigación Educativa. Abriendo puertas al conocimiento (159-178). Montevideo: Camus Ediciones. Recuperado de: http://biblioteca.clacso.edu.ar/clacso/se/20150610045455/InvestigacionEducativa.pdf.

Briz, E. (2014). Estudio de las destrezas expresivas de comunicación oral relevantes para el desempeño profesional: implicaciones sobre el currículo comunicativo. (Tesis doctoral). Departamento de las Lenguas y de las Ciencias Humanas y Sociales. Facultad de Educación. Universidad de Zaragoza. Recuperado de: https://zaguan.unizar.es/record/16929/files/TESIS-2014-094.pdf

Bronfenbrenner, U. (1987). La ecología del desarrollo humano. Experimentos en entornos naturales y diseñados. Barcelona: Paidós.

Chomsky, N. (1970). Aspectos de la teoría de la sintaxis. Madrid: Editorial Aguilar.

Gónzalez, M. C. (2010). Competencia en comunicación lingüística y audiovisual. Aula de innovación educativa, 188, 7-12.

Millet. G. (2010). Cómo se enseña la competencia en comunicación lingüística. Aula de innovación educativa, 188, 13-16.

Patton, M.Q. (1980). Qualitative evaluation and research methods. Newbury Park, CA: Sage. (ed rev.1990).

Pérez Esteve, P. (2009). La comprensión lectora y la competencia en comunicación lingüística en el nuevo marco curricular: algunas claves para su desarrollo. Educatio Siglo XXI, 1 (27), 13-32.

Pinos, M. (2012). Del aula a la vida, de la vida al aula: las competencias básicas en la escuela. Recuperado de: http://www.competenciasbasicas.net 
Popkewitz, T. S. (1989). Los valores latentes del currículo centrado en las disciplinas. En Gimeno, J. y Pérez, A. (Ed.). La enseñanza: su teoría y su práctica (306-321). Madrid: Akal.

Rebolloso, E., Fernández-Ramírez, B. y Cantón, P. (2008). Evaluación de programas de intervención social. Madrid: Síntesis.

Reyzábal, M.V. (2012). Las competencias comunicativas y lingüísticas, clave para la calidad educativa. REICE Revista Iberoamericana sobre Calidad, Eficacia y Cambio en Educación, 4 (10), 64-77.

Rossi, P. H. y Freeman, H. E. (1989). Evaluación: Un enfoque sistemático para programas sociales. México: Trillas.

Scriven, M.C. (1973). Goal Free Evaluation. En E. House (comp.): School Evaluation: The Politics and Process. Berkeley: Mac Cutchau.

Stanley, J. y Campbell, D. (1995). Diseños experimentales y cuasiexperimentales en la investigación social. Buenos Aires: Amorrortu editores.

Stake, R.E. (1975). Program evaluation, particularly responsive evaluation. Ocasional Paper Series, 5. University of Western Michigan.

Stufflebeam, D.L. y Shinkfield, A. J. (1987). Evaluación sistemática. Guía teórica y práctica. Barcelona: Paidós/MEC.

Tyler, R. (1950). Basic principle of curriculum and instruction. Chicago: Chicago University.

UNESCO (2015). Repensar l'educació. Vers un bé comú mundial? Centre UNESCO de Catalunya. Recuperado de: http://www.unescocat.org/fitxer/3685/Repensar\%201\%E2\%80\% 99educacio $\% 20 \% 28$ baixa $\% 29 . p d f$

Ley Orgánica de Educación (LOE) (Ley Orgánica 2/2006, 3 de mayo). Boletín Oficial del Estado, $\mathrm{n}^{\circ}$ 106, 2006, 4 mayo.

Ley Orgánica para la mejora de la calidad educativa (LOMCE), Ley Orgánica 8/2013, 9 de diciembre. Boletín Oficial del Estado, nº 295, 2013, 10 diciembre.

Orden ECD/65/2015, de 21 de enero, por la que se describen las relaciones entre las competencias, los contenidos y los criterios de evaluación de la educación primaria, la educación secundaria obligatoria y el bachillerato. Boletín Oficial del Estado, $\mathrm{n}^{\circ}$ 25, 2015, 29 enero.

Real Decreto 126/2014, de 28 de febrero, por el que se establece el currículo básico de la Educación Primaria. Boletín Oficial del Estado, n 52, 2014, 1 marzo. 


\section{Sobre el autor}

Autor: Martín Pinos Quílez

Institución: Centro de Innovación y Formación Educativa Juan de Lanuza (Zaragoza)

E-mail: martin.pinos@gmail.com

Información biográfica: Martín Pinos Quílez esDoctor en Ciencias de la Educación por la Universidad de Zaragoza. Máster en Aprendizaje a lo largo de la vida en contextos multiculturales. Grado en Educación Primaria y Maestro. Atesora más de 20 años de experiencia docente en la etapa de Educación Primaria y 4 cursos escolares de experiencia como asesor de formación de diseño curricular e innovación en la red de formación docente de Aragón. Actualmente trabaja como asesor de evaluación e innovación educativa en el Departamento de Educación, Cultura y Deporte del Gobierno de Aragón, y colabora, desde sus inicios (hace tres cursos), como docente del Máster de Educación Socioemocional de la Universidad de Zaragoza. Es autor de diversos libros y publicaciones relacionadas con la Educación Física y otros ámbitos educativos (competencias clave, educación en valores), tanto en editoriales privadas (Gymnos, Paidotribo...) como a través de diversas administraciones públicas (MEC, CIDE, Diputación General de Aragón, Universidad de Zaragoza...). Es primer premio nacional de innovación educativa en el año 2005, con el proyecto Un mundo de alternativas. Y es autor del Proyecto Alegría de desarrollo integrado de las competencias clave y la educación en valores. Desde 1994 colabora como ponente, de forma asidua, en acciones formativas relacionadas con el ámbito de las metodologías activas de aprendizaje, evaluación educativa y competencias clave.

ORCID: no disponible 


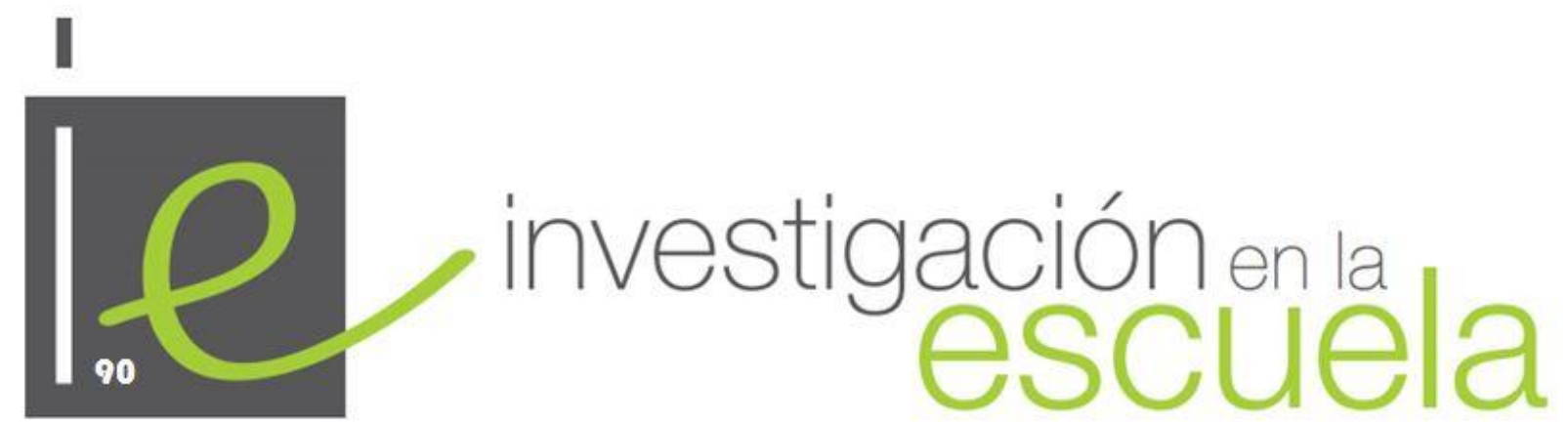

Revista académica evaluada por pares y de acceso abierto

ISSN 2443-9991

\section{(c)}

SOMERIIGHISRESERVED LOs/as lectores/as pueden copiar, mostrar, y distribuir este artículo, siempre y cuando se de crédito y atribución al autor/es y a Investigación en la Escuela, se distribuya con propósitos no-comerciales, no se altere o transforme el trabajo original. Más detalles de la licencia de Creative Commons se encuentran en http://creativecommons.org/licenses/by-nc-sa/3.0 Cualquier otro uso debe ser aprobado en conjunto por el autor/es, o Investigación en la Escuela.

Contribuya con comentarios y sugerencias en la web de la revista. Por errores y sugerencias contacte a investigacionescuela@,ddcc.uhu.es 


\section{Investigación en la escuela}

Consejo de dirección: Ana Rivero García (Universidad de Sevilla), Nicolás de Alba Fernández (Universidad de Sevilla), Pedro Cañal de León (Universidad de Sevilla), Francisco F. García Pérez (Universidad de Sevilla), Gabriel Travé González, (Universidad de Huelva), Francisco F. Pozuelos Estrada (Universidad de Huelva)

Dirección: Ana Rivero García y Nicolás de Alba Fernández

Técnico de edición: Francisco Javier López Sánchez

\section{Consejo editorial}

José Félix Angulo Rasco. Universidad de Cádiz Rosa Ma Ávila Ruiz. Universidad de Sevilla Pilar Azcárate Goded. Universidad de Cádiz Juan Bautista Martínez Rodríguez. Universidad de Granada

Nieves Blanco García. Universidad de Málaga Fernando Barragán Medero. Universidad de La Laguna José Carrillo Yáñez. Universidad de Huelva José Contreras Domingo. Universidad de Barcelona. Luis C. Contreras González. Universidad de Huelva Ana $\mathbf{M}^{\mathbf{a}}$ Criado García-Legaz. Universidad de Sevilla Rosario Cubero Pérez. Universidad de Sevilla José $\mathbf{M}^{\mathbf{a}}$ Cuenca López. Universidad de Huelva Jesús Estepa Giménez. Universidad de Huelva Rafael Feito Alonso. Universidad Complutense (Madrid)

Francisco José García Gallardo. Universidad de Huelva

Soledad García Gómez. Universidad de Sevilla J. Eduardo García Díaz. Universidad de Sevilla
Fernando Hernández Hernández. Universidad de Barcelona

Salvador Llinares Ciscar. Universidad de Alicante Alfonso Luque Lozano. Universidad de Sevilla Rosa Martín del Pozo. Universidad Complutense (Madrid)

José Martín Toscano. IES Fernando Herrera (Sevilla) Jaume Martínez Bonafé. Universidad de Valencia F. Javier Merchán Iglesias. Universidad de Sevilla Emilia Moreno Sánchez. Universidad de Huelva. Rosario Ortega Ruiz. Universidad de Córdoba Antonio de Pro Bueno. Universidad de Murcia Fco. de Paula Rodríguez Miranda. Universidad de Huelva

Pedro Sáenz-López Buñuel. Universidad de Huelva Antoni Santisteban Fernández. Universidad Autónoma (Barcelona)

Emilio Solís Ramírez. Catedrático de IES. $\mathbf{M}^{\mathbf{a}}$ Victoria Sánchez García. Universidad de Sevilla. Magdalena Suárez Ortega. Universidad de Sevilla

\section{Consejo asesor}

Manuel Area Moreira. Universidad de La Laguna

Jaume Carbonell. Director Cuadernos de Pedagogía. Barcelona

César Coll. Universidad de Barcelona

Christopher Day. Universidad de Nothingham. U.K.

Juan Delval. Universidad Nacional de Educación a Distancia

John Elliott. Universidad de East Anglia. Norwich. U.K.

José Gimeno Sacritán. Universidad de Valencia

André Giordan. Universidad de Paris VII y Ginebra

Francisco Imbernón. Universidad de Barcelona

Ángel Pérez Gómez. Universidad de Málaga

Rafael Porlán Ariza. Universidad de Sevilla

Francesco Tonucci. Instituto de Pedagogía del C.N.R. Roma

Jurjo Torres Santomé. Universidad de A Coruña 
